Discussion Paper No. 2008-9

\title{
ROBUST EXPONENTIAL HEDGING AND INDIFFERENCE VALUATION
}

\section{KeItA OWARI}

October, 2008 


\title{
ROBUST EXPONENTIAL HEDGING AND INDIFFERENCE VALUATION
}

\author{
KEITA OWARI \\ Graduate School of Economics, Hitotsubashi University \\ 2-1 Naka, Kunitachi, Tokyo 186-8601, Japan
}

\begin{abstract}
We discuss the problem of exponential hedging in the presence of model uncertainty expressed by a set of probability measures. This is a robust utility maximization problem with a contingent claim. We first consider the dual problem which is the minimization of penalized relative entropy over a product set of probability measures, showing the existence and variational characterizations of the solution. These results are applied to the primal problem. Then we consider the robust version of exponential utility indifference valuation, giving the representation of indifference price using a duality result.
\end{abstract}

\section{INTRODUCTION}

We consider the problem of exponential hedging, taken up by [7, in the presence of model uncertainty. Mathematically, this is a robust utility maximization problem with a contingent claim.

Maximization of expected utility is a classical problem in mathematical finance, initiated by R.C. Merton, and a powerful duality theory is developed by Kramkov and Schachermayer 23, 24] (see also [32, 33, for extensions to utility functions defined on $\mathbb{R}$ ). On the other hand, the notion of model uncertainty, also called the Knightian uncertainty, is recently recognized in literature. That is, the subjective probability measure, say $\mathrm{P}$, defining the expected utility is often unknown in practice. Thus it is more natural to assume that only a whole class $\mathcal{P}$ of candidate models is available, rather than $P$ exactly. This formulation of model uncertainty is sometimes called the multiple-prior approach. In this framework, the theory of utility maximization has been extended to robust utility maximization:

$$
\text { maximize } \inf _{P \in \mathcal{P}} E^{P}\left[u\left(c+G_{T}(\theta)\right)\right], \quad \text { over all } \theta \in \Theta,
$$

where $c$ is the initial capital, $\Theta$ is a suitable class of portfolio strategies, $G_{T}(\theta)$ is the total gain from the trading of $\theta$, and the functional $X \mapsto \inf _{P \in \mathcal{P}} E^{P}[u(X)]$ is called the robust utility functional associated to the utility function $u$.

In this paper, we deal with a problem of the type (1.1), but with a contingent claim $H \in L^{0}$ :

$$
\text { maximize } \inf _{P \in \mathcal{P}} E^{P}\left[1-e^{-\alpha\left(c+G_{T}(\theta)-H\right)}\right], \quad \text { over all } \theta \in \Theta .
$$

The function $1-e^{-\alpha x}$ is called the exponential utility function with the risk aversion $\alpha>0$, and we call (1.2) the robust exponential hedging problem. This problem can

E-mail address: ed061002@g.hit-u.ac.jp. 
be viewed as a combination of hedging and optimal investment for the seller of $H$, whose net terminal wealth is $c+G_{T}(\theta)-H$. When $\mathcal{P}$ is a singleton, this problem coincides with the classical exponential hedging in 7 .

As in the classical case, we first consider the dual problem of (1.2), which is the minimization of relative entropy over the Cartesian product of $\mathcal{P}$ and a set of local martingale measures. We show the existence and variational characterizations of the solution without assuming the boundedness of $H$, which partially generalize the results of [12. These results are applied to the primal problem (1.2), extending the existence and duality results of 7 to the robust case. This extension allows us to consider a valuation problem of contingent claims under uncertainty based on a robust preference, namely, the robust exponential utility indifference valuation.

We close this introduction with a brief literature review. As to the classical exponential hedging, Delbaen et al. 7 develop a general duality theory, of which our results are extensions to the robust case. In the Brownian setting, Rouge and El Karoui 31 derive the BSDE for the dual problem and give the expression of the optimal strategy in terms of the solution to the dual problem, when the claim is bounded. This result is extended by Sekine [36] to the case of unbounded claims using variational methods, and by Mania and Schweizer [25] to the general continuous semimartingale setting. Some explicit examples in Brownian settings are provided by [36] with the aid of Malliavin calculus, and by Davis [6] using PDE arguments.

There is also a vast literature for the robust utility maximization. When the utility function is defined on the half line $\mathbb{R}_{+}$, Quenez 29] deals with this problem under rather stronger assumptions, including the equivalence of all $P \in \mathcal{P}$. 29] examines the case of Brownian filtration for the logarithmic and power utility functions by means of BSDE. These results are extended by Schied and Wu 35. to more general cases where not all $P \in \mathcal{P}$ are equivalent. Hernández-Hernández and Schied [19, 20] examine the duality method of [35] in the setting of stochastic factor model with logarithmic and power utility functions, with the help of stochastic control technique. Föllmer and Gundel 12 also investigate this problem, where the existence of the so-called robust $f$-projection is proved under sufficiently general assumptions (see also Gundel [18]). For more information on robust utility maximization, see Föllmer et al. [14] and references therein.

To our knowledge, the only paper dealing with the problem of the type (1.2) is Müller [27, where the problem is considered for bounded claims in a Brownian setting with all $P \in \mathcal{P}$ being equivalent. Note that the method of [27] is based on a direct BSDE argument, and do not use the duality technique as ours.

Some more detailed information will be given when we state the results.

\section{Main Results}

In this section, we state the main results of this paper. All proofs are collected in Section 4 and 5 .

\subsection{SETUP}

Let $(\Omega, \mathcal{F}, R)$ be a complete filtered probability space equipped with a filtration $\mathbb{F}=\left(\mathcal{F}_{t}\right)_{t \in[0, T]}$ satisfying the usual condition under $R$, where $T \in(0, \infty)$ is a fixed maturity date. For simplicity of notation, we assume $\mathcal{F}=\mathcal{F}_{T}$. The probability 
measure $R$ is called the reference measure, which is different from the subjective measure in the usual sense. For any probability measures $P, Q \ll R$, the RadonNikodym density $d Q / d P$ is defined in the sense of Lebesgue decomposition:

$$
\frac{d Q}{d P}:=\frac{d Q / d R}{d P / d R} 1_{\{d P / d R>0\}}+\infty \cdot 1_{\{d P / d R=0, d Q / d R>0\}} .
$$

The price process of the tradable assets is modeled by a $d$-dimensional cádlág $R$-locally bounded semimartingale $S=\left(S^{1}, \ldots, S^{d}\right)$. In this paper, we assume the interest rate is 0 , or equivalently, $S$ is already discounted. A portfolio strategy is modeled by a $d$-dimensional predictable process $\theta=\left(\theta^{1}, \ldots, \theta^{d}\right) \in L(S)$ with $\theta_{0}=0$, where $L(S):=L(S, R)$ is the set of predictable $(S, R)$-integrable processes. Also, the gain $G(\theta)$ from the strategy $\theta$ is defined by the stochastic integral, i.e., $G(\theta):=\theta \cdot S$.

The model uncertainty is described by a set $\mathcal{P}$ of probability measures on $(\Omega, \mathcal{F})$ absolutely continuous with respect to $R$. A probability measure $Q$ on $(\Omega, \mathcal{F})$ is called an absolutely continuous (resp. equivalent) local martingale measure for $S$ if $Q \ll R$ (resp. $Q \sim R$ ) and $S$ is a local martingale under $Q$. The set of all absolutely continuous (resp. equivalent) martingale measures is denoted by $\mathcal{M}_{\text {loc }}(S)$ (resp. $\left.\mathcal{M}_{\text {loc }}^{e}(S)\right)$. By identifying each measure $Q$ with its density $d Q / d R, \mathcal{M}_{\text {loc }}(S)$ is a closed convex subset of $L^{1}(R)$, since $S$ is locally bounded (see e.g. [15]). Also, we introduce the following class of martingale measures:

$$
\mathcal{Q}_{f}:=\left\{Q \in \mathcal{M}_{\text {loc }}(S): \inf _{P \in \mathcal{P}} \mathcal{H}(Q \mid P)<\infty\right\}
$$

Here, $\mathcal{H}(Q \mid P)$ denotes the relative entropy of $Q$ w.r.t. $P$, defined by

$$
\mathcal{H}(Q \mid P):= \begin{cases}E^{P}\left[\frac{d Q}{d P} \log \frac{d Q}{d P}\right] & \text { if } Q \ll P \\ +\infty & \text { otherwise }\end{cases}
$$

Suppose we are given a set $\Theta$ of portfolio strategies and a random variable $H \in L^{0}\left(\Omega, \mathcal{F}_{T}, R\right)$, which models the contingent claim. Then our primal problem is stated as:

$$
\text { minimize } \sup _{P \in \mathcal{P}} E^{P}\left[e^{-\alpha\left(\theta \cdot S_{T}-H\right)}\right], \quad \text { over all } \theta \in \Theta,
$$

where $\alpha>0$ is a fixed constant. Note that (1.2) is equivalent to (2.1) because the constants 1 and $e^{-\alpha c}$ can be canceled out. Also, $\theta \cdot S$ is defined under each $P \in \mathcal{P}$ since $P \ll R$, hence the problem (2.1) is well-defined for every class of portfolio strategies.

The dual problem of (2.1) is formally stated as:

$$
\text { minimize } \mathcal{H}(Q \mid P)-\alpha E^{Q}[H], \quad \text { over all }(Q, P) \in \mathcal{Q}_{f} \times \mathcal{P} .
$$

The formal duality between (2.1) and (2.2) follows by taking the convex conjugate of the exponential function $e^{x}$.

We now introduce the standing assumptions of this paper:

A1: $\mathcal{P}$ is convex and $\{d P / d R: P \in \mathcal{P}\}$ is weakly compact in $L^{1}(R)$.

A2: $\mathcal{Q}_{f} \times \mathcal{M}_{l o c}^{e}(S) \neq \emptyset$.

A3: $\left\{e^{\alpha|H|} d P / d R: P \in \mathcal{P}\right\}$ is $R$-uniformly integrable and

$$
\sup _{P \in \mathcal{P}} E^{P}\left[e^{(\alpha+\varepsilon)|H|}\right]<\infty, \quad \exists \varepsilon>0 .
$$


REMARK 2.1. (a) By A1, the set $\mathcal{Q}_{f}$ is convex, although it is not closed in general. Indeed, if $Q^{1}, Q^{2} \in \mathcal{Q}_{f}$, then, for every $P^{1}, P^{2} \in \mathcal{P}$ and $\lambda \in(0,1)$, $\lambda P^{1}+(1-\lambda) P^{2} \in \mathcal{P}$ and

$$
\inf _{P \in \mathcal{P}} \mathcal{H}\left(\lambda Q^{1}+(1-\lambda) Q^{2} \mid P\right) \leq \lambda \mathcal{H}\left(Q^{1} \mid P^{1}\right)+(1-\lambda) \mathcal{H}\left(Q^{2} \mid P^{2}\right) .
$$

Taking the infimum in $P^{1}, P^{2}$, the RHS is finite since $Q^{1}, Q^{2} \in \mathcal{Q}_{f}$.

(b) A2 implies that there exists a pair $(Q, P) \in \mathcal{Q}_{f} \times \mathcal{P}$ such that $Q \sim P \sim R$ and $\mathcal{H}(Q \mid P)<\infty$. In particular, $\mathcal{P} \sim R$ in the sense that, for every $A \in \mathcal{F}$,

$$
R(A)=0 \quad \Longleftrightarrow \quad P(A)=0, \quad \forall P \in \mathcal{P} .
$$

This can be understood as a no-arbitrage condition.

(c) Under A3, $H \in L^{1}(Q)$ for all $Q \in \mathcal{Q}_{f}$ (see Corollary 4.2). In particular, $\mathcal{H}(Q \mid P)-\alpha E^{Q}[H]$ is defined for all $(Q, P) \in \mathcal{Q}_{f} \times \mathcal{P}$.

(d) Although the assumptions A1-A3 are seemingly dependent on the choice of the reference measure $R$, they are not so actually: if $R^{\prime} \sim R$, then A1-A3 remains true if we replace " $R$ " by " $R$ " " in all statements. This is trivial for A2. For A3, it suffices to note that the $R$-uniform integrability is equivalent to: $\sup _{P \in \mathcal{P}} E^{P}\left[e^{\gamma|H|}\right]<\infty$ and for any $\varepsilon>0$, there exists $\delta>0$ such that $R(A)<\delta$ implies $\sup _{P \in \mathcal{P}} E^{P}\left[e^{\gamma|H|}\right]<\varepsilon$ (see e.g. [9]). A similar argument also applies to $\mathbf{A} 1$ with the help of the Dunford-Pettis theorem [9, Theorem II.25].

(e) Finally, our setup contains that of [7] as a special case where $\mathcal{P}$ is a singleton and $H$ is bounded from below.

\subsection{Dual Problem}

We begin by stating the existence result.

Theorem 2.2. Under A1-A3, (2.2) admits a maximal solution $\left(\widehat{Q}_{H}, \widehat{P}_{H}\right) \in$ $\mathcal{Q}_{f} \times \mathcal{P}$, i.e.,

$$
\mathcal{H}\left(\widehat{Q}_{H} \mid \widehat{P}_{H}\right)-\alpha E^{\widehat{Q}_{H}}[H]=\inf _{(Q, P) \in \mathcal{Q}_{f} \times \mathcal{P}}\left(\mathcal{H}(Q \mid P)-\alpha E^{Q}[H]\right),
$$

and if $(\widetilde{Q}, \widetilde{P})$ also attains the infimum, then $\widetilde{P} \ll \widehat{P}_{H}$ and $d \widetilde{Q} / d \widetilde{P}=d \widehat{Q}_{H} / d \widehat{P}_{H}$, $\widetilde{P}$-a.s.

REMARK 2.3. (a) When $\mathcal{P}$ is a singleton, a measure transformation argument (see [7]) reduces the problem (2.2) to the minimization of relative entropy alone, which goes back to Csizár [5], and is studied by Miyahara [26], Frittelli [15], Grandits and Rheinländer [17] among others in the context of mathematical finance. However, we can not remove the penalty term $-\alpha E^{Q}[H]$ in the general case, although this measure transformation is still useful.

(b) Suppose for a moment that $H=0$. Then (2.2) is a special case of the socalled robust $f$-projection with $f(x)=x \log x$ if $x>0$, and $f(0)=0$. Theorem 2.2 is then contained in Theorem 2.6 of Föllmer and Gundel [12. When $H$ is bounded, the penalty term $Q \mapsto-\alpha E^{Q}[H]$ is continuous, hence $(Q, P) \mapsto \mathcal{H}(Q \mid P)-\alpha E^{Q}[H]$ is lower semicontinuous. Then the same proof as in [12] still works in this case. Finally, if $H$ is not bounded, we can no longer expect even that the penalty term is lower semicontinuous. However, by the uniform integrability assumption A3, we can show that $(Q, P) \mapsto \mathcal{H}(Q \mid P)-\alpha E^{Q}[H]$ is still lower semicontinuous in a suitable sense. 
REMARK 2.4. The maximal solution is unique in the sense that if $\left(Q_{H}^{1}, P_{H}^{1}\right)$ and $\left(Q_{H}^{2}, P_{H}^{2}\right)$ are two maximal solutions, then $P_{H}^{1} \sim P_{H}^{2}$ and $d Q_{H}^{1} / d P_{H}^{1}=d Q_{H}^{2} / d P_{H}^{2}$, $R$-a.s. Note that solutions to (2.2) are not unique even in this sense without maximality.

We now characterize the solutions to (2.2), by variational methods. Note that the next result is true for every solution to (2.2) without maximality.

Theorem 2.5. Suppose A1-A3 and $\left(\widehat{Q}_{H}, \widehat{P}_{H}\right) \in \mathcal{Q}_{f} \times \mathcal{P}$ is a solution to (2.2).

(a) $\widehat{Q}_{H} \sim \widehat{P}_{H}$.

(b) If $Q \in \mathcal{Q}_{f}$ and $\mathcal{H}\left(Q \mid \widehat{P}_{H}\right)<\infty$, then $\log \frac{d \widehat{Q}_{H}}{d \widehat{P}_{H}}-\alpha H \in L^{1}(Q)$ and

$$
E^{\widehat{Q}_{H}}\left[\log \frac{d \widehat{Q}_{H}}{d \widehat{P}_{H}}-\alpha H\right] \leq E^{Q}\left[\log \frac{d \widehat{Q}_{H}}{d \widehat{P}_{H}}-\alpha H\right] .
$$

(c) For every $P \in \mathcal{P}$,

$$
E^{P}\left[\frac{d \widehat{Q}_{H}}{d \widehat{P}_{H}}\right] \leq E^{\widehat{P}_{H}}\left[\frac{d \widehat{Q}_{H}}{d \widehat{P}_{H}}\right]=1 .
$$

REMARK 2.6. Some related results are found. When $H=0$, then parts (b) and (c) are contained in Lemma 3.12 of [12, although an additional assumption is required there for part (a). Note that $\widehat{Q}_{H}$ is also a solution to (2.2) with $\mathcal{P}$ replaced by $\left\{\widehat{P}_{H}\right\}$. Thus if we consider the three results above separately, the variational inequality (b) follows essentially from Proposition 7.2 of Goll and Rüschendorf [16]. A similar remark applies to part (c). However, we will prove three results jointly by showing the joint variational inequality for (2.2). In particular, part (a) follows without additional assumptions.

By the above remark, the same argument as in Theorem 7.1 of [16], based on a Hahn-Banach argument and Yor's theorem [38, shows the following expression of the density $d \widehat{Q}_{H} / d \widehat{P}_{H}$.

COROLLARY 2.7. There exists a predictable process $\hat{\theta} \in L_{\text {loc }}^{1}\left(S, \widehat{Q}_{H}\right)$ such that

$$
\frac{d \widehat{Q}_{H}}{d \widehat{P}_{H}}=\widehat{c} \cdot e^{-\alpha\left(\hat{\theta} \cdot S_{T}-H\right)}, \quad \widehat{Q}_{H}-\text { a.s. },
$$

where $\widehat{c}=E^{\widehat{P}}\left[e^{-\alpha\left(\hat{\theta} \cdot S_{T}-H\right)}\right]$, and $\hat{\theta} \cdot S$ is a $\widehat{Q}_{H}$-martingale.

\subsection{Solution to the Primal Problem}

We are in the position to state the results on the primal problem (2.1), based on the results in the previous subsection. In what follows in this section, we fix a maximal solution $\left(\widehat{Q}_{H}, \widehat{P}_{H}\right) \in \mathcal{Q}_{f} \times \mathcal{P}$ to the problem (2.2).

First, we have to specify the set $\Theta$ of admissible portfolio strategies. In this paper, we consider two choices of $\Theta$, both of which result the same optimal value. The first choice is:

$$
\Theta_{b}:=\left\{\theta \in L(S): \theta_{0}=0, \theta \cdot S \text { is bounded uniformly in }(t, \omega)\right\} .
$$

This class is universal in that it depends neither on $H$ nor on $\mathcal{P}$. In particular, $\Theta_{b}$ is a priori well-defined, and if $\theta \in \Theta_{b}$, the stochastic integral $\theta \cdot S$ is a martingale under every $Q \in \mathcal{M}_{\text {loc }}(S)$, by Proposition 3.3 of Ansel and Stricker [1. For $\Theta_{b}$, we can compute the optimal value of the problem by the duality equality: 
TheOREM 2.8. Under $\boldsymbol{A} \mathbf{1}-\boldsymbol{A} 3$, it holds that

$$
\inf _{\theta \in \Theta_{b}} \sup _{P \in \mathcal{P}} E^{P}\left[e^{-\alpha\left(\theta \cdot S_{T}-H\right)}\right]=e^{-\inf _{(Q, P) \in \mathcal{Q}_{f} \times \mathcal{P}}\left(\mathcal{H}(Q \mid P)-\alpha E^{Q}[H]\right)} .
$$

Note that the infimum in the LHS is not attained in $\Theta_{b}$ even in the case where $\mathcal{P}$ is a singleton, $H=0$ and $S$ is a geometric Brownian motion. However, the universality of $\Theta_{b}$ is important for application, especially for utility indifference valuation to be discussed in the next section.

If we want to get an optimizer, we need to enlarge the class $\Theta_{b}$ to:

$$
\Theta_{H}:=\left\{\theta \in L(S): \theta_{0}=0, \theta \cdot S \text { is a martingale under } \forall Q \in \mathcal{Q}_{f}\left(\widehat{P}_{H}\right)\right\},
$$

where $\mathcal{Q}_{f}\left(\widehat{P}_{H}\right):=\left\{Q \in \mathcal{Q}_{f}: \mathcal{H}\left(Q \mid \widehat{P}_{H}\right)<\infty\right\}$. Obviously, this class depends on $\widehat{P}_{H}$ in general. In particular, the admissibility of a strategy depends on the claim, and $\Theta_{H}$ is not known to us until we solve the dual problem. However, in some special cases, $\Theta_{H}$ coincides with an intermediate class:

$$
\Theta_{\mathcal{P}}:=\left\{\theta \in L(S): \theta_{0}=0, \theta \cdot S \text { is a martingale under } \forall Q \in \mathcal{Q}_{f}\right\} .
$$

Note that $\Theta_{b} \subset \Theta_{\mathcal{P}} \subset \Theta_{H}$, and $\Theta_{\mathcal{P}}$ is universal in the above sense. Also, we need an additional assumption:

A4: $\widehat{Q}_{H} \sim \widehat{P}_{H} \sim R$.

Note that $\widehat{Q}_{H}$ and $\widehat{P}_{H}$ are always equivalent by Theorem 2.5 (a). However, $\widehat{P}_{H}$ may fail to be equivalent with the reference measure $R$ in general. A4 is trivially satisfied if we assume that all elements of $\mathcal{P}$ are equivalent.

TheOREM 2.9. (a) Under $\boldsymbol{A} 1$-A3, we have

$$
\inf _{\theta \in \Theta_{H}} \sup _{P \in \mathcal{P}} E^{P}\left[e^{-\alpha\left(\theta \cdot S_{T}-H\right)}\right]=e^{-\inf _{(Q, P) \in \mathcal{Q}_{f} \times \mathcal{P}}\left(\mathcal{H}(Q \mid P)-\alpha E^{Q}[H]\right)} .
$$

(b) If in addition we assume $\boldsymbol{A} 4$, then $\hat{\theta}$ appearing in (2.8) is in $\Theta_{H}$ and the pair $\left(\hat{\theta}, \widehat{P}_{H}\right)$ is a saddle point of the map $(\theta, P) \mapsto E^{P}\left[e^{-\alpha\left(\theta \cdot S_{T}-H\right)}\right]$ on $\Theta_{H} \times \mathcal{P}$. In particular, (i) $\hat{\theta}$ is a solution to (2.1) with $\Theta=\Theta_{H}$, (ii) $\widehat{P}_{H}$ is a least favorable measure in that

$$
\inf _{\theta \in \Theta_{H}} E^{\widehat{P}_{H}}\left[e^{-\alpha\left(\theta \cdot S_{T}-H\right)}\right]=\sup _{P \in \mathcal{P}} \inf _{\theta \in \Theta_{H}} E^{P}\left[e^{-\alpha\left(\theta \cdot S_{T}-H\right)}\right],
$$

and (iii) the minimax equality holds:

$$
\inf _{\theta \in \Theta_{H}} \sup _{P \in \mathcal{P}} E^{P}\left[e^{-\alpha\left(\theta \cdot S_{T}-H\right)}\right]=\sup _{P \in \mathcal{P}} \inf _{\theta \in \Theta_{H}} E^{P}\left[e^{-\alpha\left(\theta \cdot S_{T}-H\right)}\right] .
$$

In part (b) above, the solution is unique in that if $\tilde{\theta}$ is another solution, then $\tilde{\theta} \cdot S=\hat{\theta} \cdot S$ up to $R$-indistinguishability. This follows from the uniqueness of maximal solution to (2.2) in the sense of Remark 2.4

REMARK 2.10. As pointed out in the classical case by Schachermayer 32, 33, the choice of admissible strategies for utility maximization problems, both in the subjective and robust cases, is a delicate issue if the effective domain of the utility function $u$ is the whole $\mathbb{R}$ as in our case: $u(x)=1-e^{-\alpha x}$. When $u$ is defined on $\mathbb{R}_{+}$with the so-called Inada condition, i.e., $u^{\prime}(0)=+\infty$ and $u^{\prime}(\infty)=0$, a suitable choice is

$$
\Theta_{c}:=\left\{\theta \in L(S): \theta_{0}=0, c+\theta \cdot S \geq 0\right\},
$$

for each initial capital $c$. Since each wealth process $c+\theta \cdot S$ is bounded from below, it is a super martingale under each $Q \in \mathcal{M}_{\text {loc }}(S)$, hence $\Theta_{c}$ is free of arbitrage. 
Moreover, the existence of optimizer and the duality equality as (2.10) can be proved for $\Theta_{c}$ under sufficiently general assumptions. See [35] for detail. When $u$ is defined on the whole $\mathbb{R}$, it seems natural to consider

$$
\Theta_{b b}:=\left\{\theta \in L(S): \theta_{0}=0, \theta \cdot S \text { is bounded from below }\right\} .
$$

However, this class is not large enough to admit an optimizer.

Our choice $\Theta_{b}$ (resp. $\Theta_{H}$ ) corresponds to $\Theta_{3}$ (resp. $\Theta_{H}$ ) of [7]. $\Theta_{b}$ (and $\Theta_{H}$ under A4) is arbitrage-free in that, if $\theta \in \Theta_{b}$ satisfies $P\left(\theta \cdot S_{T} \geq 0\right)=1$ for all $P \in \mathcal{P}$, then $P\left(\theta \cdot S_{T}=0\right)=1$ for all $P \in \mathcal{P}$.

Finally, in the proof of Theorem 2.9, we actually use only the fact that $\Theta_{b} \subset \Theta_{H}$ and $\theta \cdot S$ is a super martingale under $\widehat{Q}_{H}$ for each $\theta \in \Theta_{H}$. In particular, this theorem remains true if we replace $\Theta_{H}$ by another $\Theta$ whenever $\Theta_{b} \cup\{\hat{\theta}\} \subset \Theta \subset \Theta_{H, 0}^{\prime}$, where $\Theta_{H, 0}^{\prime}$ is the set of portfolio strategies whose wealths are $\widehat{Q}_{H}$-supermartingale, which corresponds to $\Theta_{1}$ of [7].

REMARK 2.11. Theorem 2.8 (resp. 2.9) is an extension of Theorem 2.3 (resp. 2.2) of [7] (see also Kabanov and Stricker [22]) to the robust case. Also, similar duality results are available in robust utility maximization. When the utility function $u$ is defined on $\mathbb{R}_{+}$and $H=0$, Schied and $\mathrm{Wu}$ [35] prove the duality, as our Theorem 2.8, and some additional properties of value functions, on which our idea of the proof of Theorem 2.8 is based. Schied [34] extends the argument in 35] to the case with penalized robust utilities.

\section{Application to Indifference Valuation}

In this section, we consider a valuation problem of contingent claims under model uncertainty based on the results of the previous section, namely, the robust exponential utility indifference valuation. This is a robust version of the exponential utility indifference valuation studied by Rouge and El Karoui [31, Mania and Schweizer 25] and others.

Recall that the robust exponential utility functional is defined by

$$
U(X):=\inf _{P \in \mathcal{P}} E^{P}\left[1-e^{-\alpha X}\right], \quad X \in L^{0}(R) .
$$

DEFinition 3.1. For a contingent claim $H$, we define the robust exponential utility indifference price as a unique real number $p_{\alpha}(H)$ satisfying:

$$
\sup _{\theta \in \Theta_{b}} U\left(p_{\alpha}(H)+\theta \cdot S_{T}-H\right)=\sup _{\theta \in \Theta_{b}} U\left(\theta \cdot S_{T}\right) .
$$

Here we use $\Theta_{b}$ as admissible strategies for universality of the definition. Although the infimums in both sides of (3.2) are not attained, it does not cause any problem for our purpose. Let $\left(\widehat{Q}_{0}, \widehat{P}_{0}\right) \in \mathcal{Q}_{f} \times \mathcal{P}$ be a maximal solution to the problem (2.2) with $H \equiv 0$, whose existence is guaranteed by (2.5). Then Theorem 2.8 immediately gives the following expression of $p_{\alpha}(H)$.

Corollary 3.2. Under A1-A3,

$$
p_{\alpha}(H)=\sup _{Q \in \mathcal{Q}_{f}}\left(E^{Q}[H]-\gamma_{\alpha}(Q)\right),
$$

where $\gamma$ is a penalty function defined by

$$
\gamma_{\alpha}(Q)=\frac{1}{\alpha}\left(\inf _{P \in \mathcal{P}} \mathcal{H}(Q \mid P)-\mathcal{H}\left(\widehat{Q}_{0} \mid \widehat{P}_{0}\right)\right) .
$$


Proof. For every $p \in \mathbb{R}, \theta \in \Theta_{b}$ and $H \in L^{0}(R)$,

$$
U\left(p+\theta \cdot S_{T}-H\right)=1-e^{-\alpha p} \sup _{P \in \mathcal{P}} E^{P}\left[e^{-\alpha\left(\theta \cdot S_{T}-H\right)}\right] .
$$

by definition. Thus under A1-A3, Theorem 2.8 yields that

$$
\begin{aligned}
\sup _{\theta \in \Theta_{b}} U\left(p+\theta \cdot S_{T}-H\right) & =1-\inf _{\theta \in \Theta_{b}} \sup _{P \in \mathcal{P}} E^{P}\left[e^{-\alpha\left(\theta \cdot S_{T}-H\right)}\right] \\
& =1-e^{-\alpha p-\inf _{(Q, P) \in \mathcal{Q}_{f} \times \mathcal{P}}\left(\mathcal{H}(Q \mid P)-\alpha E^{Q}[H]\right)} .
\end{aligned}
$$

Noting that $H \equiv 0$ trivially satisfies $\mathbf{A 3}$, a similar computation yields that

$$
\sup _{\theta \in \Theta_{b}} U\left(\theta \cdot S_{T}\right)=1-e^{-\inf _{(Q, P) \in \mathcal{Q}_{f} \times \mathcal{P}} \mathcal{H}(Q \mid P)} .
$$

Comparing (3.5), (3.6) and (3.7), we have

$$
\begin{aligned}
p_{\alpha}(H) & =\sup _{Q \in \mathcal{Q}_{f}}\left(E^{Q}[H]-\frac{1}{\alpha} \inf _{P \in \mathcal{P}} \mathcal{H}(Q \mid P)\right)-\inf _{(Q, P) \in \mathcal{Q}_{f} \times \mathcal{P}} \mathcal{H}(Q \mid P) \\
& =\sup _{Q \in \mathcal{Q}_{f}}\left(E^{Q}[H]-\gamma_{\alpha}(Q)\right) .
\end{aligned}
$$

REMARK 3.3. (a) The concept of utility indifference valuation is quite popular, which goes back to Hodges and Neuberger 21. When $\mathcal{P}$ is a singleton, the expression (3.3) is shown by Rouge and El Karoui 31 for bounded $H$ in a Brownian setting, and by $[7$ and Becherer [3] for general semimartingale setting. Also, Mania and Schweizer [25] study a dynamic version when the filtration is continuous. An explicit example in a diffusion setting is provided by Musiela and Zariphopoulou 28.

(b) (3.3) is a mathematically minor extension of these results to the robust case. However, from Economic point of view, this valuation procedure explicitly takes model uncertainty into account. To our knowledge, there are few research on valuation under model uncertainty, excepting superreplication prices in the uncertain volatility model by Avellaneda et al. 2] and Denis and Martini [10].

Note that Corollary 4.2 applies to all bounded claims. If we consider $p_{\alpha}$ as a function on $L^{\infty}$, the following properties are straightforward.

(a) $p_{\alpha}$ is convex.

(b) $p_{\alpha}(H+c)=p_{\alpha}(H)+a$ for all $H \in L^{\infty}$ and $c \in \mathbb{R}$.

(c) $H \leq H^{\prime}$ implies $p_{\alpha}(H) \leq p_{\alpha}\left(H^{\prime}\right)$.

(d) $-\|H\|_{\infty} \leq \inf _{Q \in \mathcal{Q}_{f}} E^{Q}[H] \leq p_{\alpha}(H) \leq \sup _{Q \in \mathcal{Q}_{f}} E^{Q}[H] \leq\|H\|_{\infty}$.

In particular, $\rho_{\alpha}(X):=p_{\alpha}(-X)$ defines a convex risk measure in the sense of Föllmer and Schied [13], and $p_{\alpha}(H)$ is interpreted as the risk of payment $-H$ for the option writer whose preference is given by the robust exponential utility. Also, $p_{\alpha}(H)$ can be understood as a price of $H$ in view of (d).

\section{Proofs of Theorem 2.2 and 2.5}

Note first that we can assume without loss of generality that $\alpha=1$, by replacing $H$ by $\alpha H$. Then the assumption $\mathbf{A} \mathbf{3}$ is written as

A3': $\left\{e^{|H|}(d P / d R): P \in \mathcal{P}\right\}$ is uniformly integrable and

$$
\sup _{P \in \mathcal{P}} E\left[e^{(1+\varepsilon)|H|}\right]<\infty, \quad \text { for } \exists \varepsilon>0 .
$$




\subsection{Proof of Theorem 2.2}

We need some preparations. The next lemma is found in Delbaen et al. [7, and follows from the fact that the convex conjugate of the function $f(x)=x \log x$ is $f^{*}(y)=e^{-1} e^{y}$.

Lemma 4.1 ([7, Lemma 3.5). For any pair $(Q, P)$ of probability measures on $(\Omega, \mathcal{F})$ and a random variable $B$,

$$
E^{Q}[|B|] \leq \mathcal{H}(Q \mid P)+e^{-1} E^{P}\left[e^{|B|}\right] .
$$

Corollary 4.2. Under $\boldsymbol{A}^{\prime}$ ', $H \in L^{1}(Q)$ for each $Q \in \mathcal{Q}_{f}$.

Proof. If $Q \in \mathcal{Q}_{f}$, there exists $P \in \mathcal{P}$ with $\mathcal{H}(Q \mid P)<\infty$ by definition. Then (4.2) together with $\mathbf{A} 3$ ' yield the desired result.

Lemma 4.3. Under A3', there exists constants $C_{1}, C_{2}>0$ depending only on $H$ and $\mathcal{P}$ such that

$$
\mathcal{H}(Q \mid P) \leq C_{1}+C_{2}\left(\mathcal{H}(Q \mid P)-E^{Q}[H]\right), \quad \forall(Q, P) \in \mathcal{Q}_{f} \times \mathcal{P} .
$$

In particular,

$$
\mathcal{H}(Q \mid P)-E^{Q}[H] \geq-\frac{C_{1}}{C_{2}}>-\infty, \quad \forall(Q, P) \in \mathcal{Q}_{f} \times \mathcal{P}
$$

Proof. Note first that, if $Q \in \mathcal{Q}_{f}, P \in \mathcal{P}$ and $\mathcal{H}(Q \mid P)=+\infty$, then the both sides of (4.3) are $+\infty$ for any positive constants $C_{1}, C_{2}$, since $H \in L^{1}(Q)$ by Corollary 4.2. Hence it suffices to consider the case $Q \ll P$.

We fix $\varepsilon>0$ satisfying (4.1). Then for any $Q \in \mathcal{Q}_{f}$ and $P \in \mathcal{P}$ with $\mathcal{H}(Q \mid P)<$ $\infty$, Lemma 4.1 applied to $B=(1+\varepsilon) H$ shows that

$$
\begin{aligned}
-E^{Q}[H] & \geq-\frac{1}{(1+\varepsilon)}\left(\mathcal{H}(Q \mid P)+e^{-1} E^{P}\left[e^{(1+\varepsilon)|H|}\right]\right) \\
& \geq-\frac{1}{(1+\varepsilon)}\left(\mathcal{H}(Q \mid P)+e^{-1} \sup _{P \in \mathcal{P}} E^{P}\left[e^{(1+\varepsilon)|H|}\right]\right) .
\end{aligned}
$$

Here $\sup _{P \in \mathcal{P}} E^{P}\left[e^{\gamma|H|}\right]<\infty$ by A3'. Adding $\mathcal{H}(Q \mid P)$ to both sides,

$$
\mathcal{H}(Q \mid P)-E^{Q}[H] \geq \frac{\varepsilon}{1+\varepsilon} \mathcal{H}(Q \mid P)-\frac{e^{-1}}{1+\varepsilon} \sup _{P \in \mathcal{P}} E^{P}\left[e^{\gamma|H|}\right] .
$$

Hence we get (4.3) by taking

$$
C_{1}=\frac{e^{-1}}{\varepsilon} \sup _{P \in \mathcal{P}} E^{P}\left[e^{\gamma|H|}\right], \quad C_{2}=\frac{1+\varepsilon}{\varepsilon} .
$$

(4.4) follows from (4.3) since $\mathcal{H}(Q \mid P) \geq 0$ for every pair $(Q, P)$ of probability measures.

REMARK 4.4. A related estimate is found in Bordigoni et al. 4], where robust utility maximization problem with penalized robust utility is investigated using a direct BSDE method for continuous semimartingales. Corollary 4.2 and Lemma 4.3 shows that the problem (2.2) is well-defined.

The next result is a restatement of Föllmer and Gundel [12], Lemma 2.12. 
Lemma 4.5. Suppose $\boldsymbol{A} 1$. Let $\mathcal{Q}$ be a set of probability measures on $(\Omega, \mathcal{F})$, which are absolutely continuous w.r.t. $R$. If

$$
\sup _{Q \in \mathcal{Q}} \inf _{P \in \mathcal{P}} \mathcal{H}(Q \mid P)<\infty
$$

then $\{d Q / d R: Q \in \mathcal{Q}\}$ is uniformly integrable.

ProOF. Note that $\mathcal{H}(\cdot \mid \cdot)$ is a $f$-divergence with $f(x)=x \log x$ and $\lim _{x} \nearrow \infty \frac{f(x)}{x}=$ $+\infty$. This together with A1 allows us to apply Lemma 2.12 of [12] to show the existence of a function $l:[0, \infty) \longrightarrow[0, \infty)$ such that $\lim _{x} / \infty \frac{l(x)}{x}=\infty$ and

$$
\forall c \in \mathbb{R}_{+}, \quad \exists c_{0} \in \mathbb{R}_{+} \quad \text { s.t. } \quad \inf _{P \in \mathcal{P}} \mathcal{H}(Q \mid P) \leq c \quad \Longrightarrow \quad E^{R}[l(d Q / d R)] \leq c_{0} .
$$

Taking $c=\sup _{Q \in \mathcal{Q}} \inf _{P \in \mathcal{P}} \mathcal{H}(Q \mid P)$, we have $\sup _{Q \in \mathcal{Q}} E^{R}[l(d Q / d R)]<\infty$. The result then follows from the de la Vallée-Poussion criteria.

Note that the map $(Q, P) \mapsto \mathcal{H}(Q \mid P)$ is lower semicontinuous in that if $d Q^{n} / d R \longrightarrow$ $d Q / d R$ and $d P^{n} / d R \longrightarrow d P / d R$ a.s., then $\mathcal{H}(Q \mid P) \leq \liminf _{n \rightarrow \infty} \mathcal{H}\left(Q^{n} \mid P^{n}\right)$. But we need a slightly more general result.

Lemma 4.6. Suppose A3'. Let $\left\{\left(Q^{n}, P^{n}\right)\right\}_{n \in \mathbb{N}}$ be a sequence in $\mathcal{Q}_{f} \times \mathcal{P}$. If there exists $(Q, P) \in \mathcal{Q}_{f} \times \mathcal{P}$ such that $\mathcal{H}(Q \mid P)<\infty$ and $d Q^{n} / d R$ (resp. $\left.d P^{n} / d R\right)$ converges a.s. to $d Q / d R$ (resp. $d P / d R$ ), then

$$
\mathcal{H}(Q \mid P)-E^{Q}[H] \leq \liminf _{n \rightarrow \infty}\left(\mathcal{H}\left(Q^{n} \mid P^{n}\right)-E^{Q^{n}}[H]\right) .
$$

Proof. Define $\widetilde{P}^{n}$ and $\widetilde{P}$ by

$$
\frac{d \widetilde{P}^{n}}{d R}:=\frac{e^{H}}{E^{P^{n}}\left[e^{H}\right]} \frac{d P^{n}}{d R}, \quad(n \in \mathbb{N}) \text { and } \quad \frac{d \widetilde{P}}{d R}:=\frac{e^{H}}{E^{P}\left[e^{H}\right]} \frac{d P}{d R} .
$$

By A3', $\left\{e^{H} d P^{n} / d R\right\}_{n}$ is uniformly integrable, hence $\lim _{n \rightarrow \infty} E^{P^{n}}\left[e^{H}\right]=E^{P}\left[e^{H}\right]$ and $d \widetilde{P}^{n} / d R \longrightarrow d \widetilde{P} / d R$, a.s. and in $L^{1}(R)$. In particular, we have $\mathcal{H}(Q \mid \widetilde{P}) \leq$ $\liminf _{n \rightarrow \infty} \mathcal{H}\left(Q^{n} \mid \widetilde{P}^{n}\right)$. Noting that

$$
\begin{aligned}
\mathcal{H}\left(Q^{n} \mid P^{n}\right)-E^{Q^{n}}[H] & =E^{Q^{n}}\left[\log \frac{d Q^{n}}{d \widetilde{P}^{n}}\right]-\log E^{P^{n}}\left[e^{H}\right] \\
& =\mathcal{H}\left(Q^{n} \mid \widetilde{P}^{n}\right)-\log E^{P^{n}}\left[e^{H}\right],
\end{aligned}
$$

we have

$$
\begin{aligned}
\mathcal{H}(Q \mid P)-E^{Q}[H] & =\mathcal{H}(Q \mid \widetilde{P})-\log E^{P}\left[e^{H}\right] \\
& \leq \liminf _{n \rightarrow \infty}\left(\mathcal{H}\left(Q^{n} \mid \widetilde{P}^{n}\right)-\log E^{P^{n}}\left[e^{H}\right]\right) \\
& =\liminf _{n \rightarrow \infty}\left(\mathcal{H}\left(Q^{n} \mid P^{n}\right)-E^{Q^{n}}[H]\right) .
\end{aligned}
$$

We proceed to the proof of Theorem 2.2. We first prove the existence of a (not necessarily maximal) solution. Then we construct a maximal solution.

Proof of Existence. We claim that there exists $(\widehat{Q}, \widehat{P}) \in \mathcal{Q}_{f} \times \mathcal{P}$ such that

$$
\mathcal{H}(\widehat{Q} \mid \widehat{P})-E^{\widehat{Q}}[H]=\inf _{(Q, P) \in \mathcal{Q}_{f} \times \mathcal{P}}\left(\mathcal{H}(Q \mid P)-E^{Q}[H]\right)=: a .
$$


Note that $-\infty<a<+\infty$ by Lemma 4.3 and A2. Let $\left\{\left(Q^{n}, P^{n}\right)\right\}_{n \in \mathbb{N}} \subset \mathcal{Q}_{f} \times \mathcal{P}$ be such that

$$
\mathcal{H}\left(Q^{n} \mid P^{n}\right)-E^{Q^{n}}[H] \searrow a \quad(n \longrightarrow \infty) .
$$

We denote the density of $Q^{n}$ (resp. $P^{n}$ ) w.r.t. $R$ by $Z^{n}$ (resp. $D^{n}$ ) for each $n$.

Applying twice the Komlos theorem ([8, Theorem A.1) (first to the sequence $\left\{Z^{n}\right\}$ and then to $\left.\left\{D^{n}\right\}\right)$, we get another sequence $\left\{\left(\widehat{Z}^{n}, \widehat{D}^{n}\right)\right\}$ such that

$$
\widehat{Z}^{n} \longrightarrow \widehat{Z}, \quad \widehat{D}^{n} \longrightarrow \widehat{D}, \quad R \text {-a.s. }
$$

for some positive random variables $\widehat{Z}$ and $\widehat{D}$, and

$$
\left(\widehat{Z}^{n}, \widehat{D}^{n}\right) \in \operatorname{conv}\left(\left(Z^{n}, D^{n}\right),\left(Z^{n+1}, D^{n+1}\right), \ldots\right) .
$$

Since $\mathcal{Q}_{f}$ and $\mathcal{P}$ are convex and each $\widehat{Z}^{n}$ (resp. $\widehat{D}^{n}$ ) is a convex combination of $\left\{d Q^{n} / d R\right\}$ (resp. $\left.\left\{d P^{n} / d R\right\}\right)$, there exists an element $\widehat{Q}^{n} \in \mathcal{Q}_{f}$ (resp. $\widehat{P}^{n} \in \mathcal{P}$ ) such that $\widehat{Z}^{n}=\widehat{Q}^{n} / d R$ (resp. $\widehat{D}^{n}=d \widehat{P}^{n} / d R$ ). By the construction of original sequence $\left\{\left(Q^{n}, P^{n}\right)\right\}$, we see that

$$
a \leq \mathcal{H}\left(\widehat{Q}^{n} \mid \widehat{P}^{n}\right)-E^{\widehat{Q}^{n}}[H] \leq \mathcal{H}\left(Q^{n} \mid P^{n}\right)-E^{Q^{n}}[H] \longrightarrow a .
$$

By A1, the sequence $\left\{\widehat{D}^{n}\right\}_{n \in \mathbb{N}}$ is uniformly integrable, hence the convergence $\widehat{D}^{n} \longrightarrow \widehat{D}$ takes place in $L^{1}(R)$ and there exists a probability measure $\widehat{P} \in \mathcal{P}$ with $d \widehat{P} / d R=\widehat{D}$. Also, by Lemma 4.3 and the construction of the sequence $\left\{\left(\widehat{Q}^{n}, \widehat{P}^{n}\right)\right\}$, we see that

$$
\begin{aligned}
\mathcal{H}\left(\widehat{Q}^{n} \mid \widehat{P}^{n}\right) & \leq C_{1}+C_{2}\left(\mathcal{H}\left(\widehat{Q}^{n} \mid \widehat{P}^{n}\right)-E^{\widehat{Q}^{n}}[H]\right) \\
& \leq C_{1}+C_{2}\left(\mathcal{H}\left(Q^{n} \mid P^{n}\right)-E^{Q^{n}}[H]\right) \\
& \leq C_{1}+C_{2}\left(\mathcal{H}\left(Q^{1} \mid P^{1}\right)-E^{Q^{1}}[H]\right),
\end{aligned}
$$

for each $n$. In particular, $\sup _{n} \inf _{P \in \mathcal{P}} \mathcal{H}\left(\widehat{Q}^{n} \mid P\right)<\infty$ and Lemma 4.5 implies that $\left\{d \widehat{Q}^{n} / d R\right\}_{n}$ is uniformly integrable. Hence the convergence $d \widehat{Q}^{n} / d R \longrightarrow \widehat{Z}$ also takes place in $L^{1}(R)$ and there exists $\widehat{Q} \in \mathcal{M}_{\text {loc }}(S)$ such that $d \widehat{Q} / d R=\widehat{Z}$ by the closedness of $\mathcal{M}_{\text {loc }}(S)$. Then we have $\mathcal{H}(\widehat{Q} \mid \widehat{P}) \leq \liminf _{n \rightarrow \infty} \mathcal{H}\left(\widehat{Q}^{n} \mid \widehat{P}^{n}\right)<\infty$ by (4.7). Therefore, $\widehat{Q} \in \mathcal{Q}_{f}$, and Lemma 4.6 shows that

$$
\mathcal{H}(\widehat{Q} \mid \widehat{P})-E^{\widehat{Q}}[H] \leq \liminf _{n \rightarrow \infty}\left(\mathcal{H}\left(\widehat{Q}^{n} \mid \widehat{P}^{n}\right)-E^{\widehat{Q}^{n}}[H]\right)=a .
$$

Hence $(\widehat{Q}, \widehat{P})$ is a desired pair.

For the construction of a maximal solution, we need a lemma. Let $\mathcal{S}$ be the set of solutions to (2.2), i.e.,

$$
\mathcal{S}:=\left\{(Q, P) \in \mathcal{Q}_{f} \times \mathcal{P}:(Q, P) \text { satisfies (4.6) }\right\} .
$$

LEMMA 4.7. (a) $\mathcal{S}$ is countably convex in the sense that, if $\left(Q^{n}, P^{n}\right) \in \mathcal{S}$, $a_{n}>0$ for each $n$ and $\sum_{n \in \mathbb{N}} a_{n}=1$, then $\left(\sum_{n} a_{n} Q^{n}, \sum_{n} a_{n} P^{n}\right) \in \mathcal{S}$.

(b) If $\left(Q^{0}, P^{0}\right),\left(Q^{1}, P^{1}\right) \in \mathcal{S}$ and $P^{0} \ll P^{1}$, then $d Q^{0} / d P^{0}=d Q^{1} / d P^{1}, P^{0}$-a.s.

Proof of Lemma. (a) First, $\mathcal{S}$ is convex by the convexity of $(Q, P) \mapsto \mathcal{H}(Q \mid P)-$ $E^{Q}[H]$. Let $\left(Q^{n}, P^{n}\right) \in \mathcal{S}, a_{n}>0$ for each $n$ and $\sum_{n} a_{n}=1$. Since $Q^{n}$ and $P^{n}$ are probability measures for each $n$, the serieses

$$
\sum_{n} a_{n} \frac{d Q^{n}}{d R}, \quad \sum_{n} a_{n} \frac{d P^{n}}{d R}
$$


are absolutely convergent in $L^{1}(R)$. Hence $(\widetilde{Q}, \widetilde{P}):=\left(\sum_{n} a_{n} Q^{n}, \sum_{n} a_{n} P^{n}\right)$ is welldefined as a pair of probability measures, and $(\widetilde{Q}, \widetilde{P}) \in \mathcal{M}_{l o c}(S) \times \mathcal{P}$ by closedness. Setting

$$
\widetilde{Q}^{n}:=\frac{1}{\sum_{k=1}^{n} a_{k}} \sum_{k=1}^{n} a_{k} Q^{k}, \quad \widetilde{P}^{n}:=\frac{1}{\sum_{k=1}^{n} a_{k}} \sum_{k=1}^{n} a_{k} P^{k}, \quad \forall n,
$$

$\left(\widetilde{Q}^{n}, \widetilde{P}^{n}\right) \in \mathcal{S}$ by convexity, and $d \widetilde{Q}^{n} / d R \longrightarrow d \widetilde{Q} / d R, d \widetilde{P}^{n} / d R \longrightarrow d \widetilde{P} / d R$, a.s. and in $L^{1}(R)$. Using the same computation as in (4.7) and the fact that each $\left(\widetilde{Q}^{n}, \widetilde{P}^{n}\right)$ is optimal, we have $\mathcal{H}(\widetilde{Q} \mid \widetilde{P})<\infty$. Then Lemma 4.6 conclude the proof.

(b) Let $\left(Q^{0}, P^{0}\right),\left(Q^{1}, P^{1}\right) \in \mathcal{S}$ and define $Q_{\lambda}:=\lambda Q^{1}+(1-\lambda) Q^{0}, P_{\lambda}:=\lambda P^{1}+$ $(1-\lambda) P^{0}$ for each $\lambda \in[0,1]$. Then for each $\lambda$,

$$
\lambda \frac{d P^{1}}{d P_{\lambda}}+(1-\lambda) \frac{d P^{0}}{d P_{\lambda}}=1
$$

and

$$
\lambda \frac{d P^{1}}{d P_{\lambda}} \frac{d Q^{1}}{d P^{1}}+(1-\lambda) \frac{d P^{0}}{d P_{\lambda}} \frac{d Q^{0}}{d P_{0}}=\lambda \frac{d Q^{1}}{d P_{\lambda}}+(1-\lambda) \frac{d Q^{0}}{d P_{\lambda}}=\frac{d Q_{\lambda}}{d P_{\lambda}} .
$$

Also, we set $f(x)=x \log x$ for simplicity of notation. Then

$$
\begin{aligned}
\mathcal{H}\left(Q_{\lambda} \mid P_{\lambda}\right) & -E^{Q_{\lambda}}[H] \\
& \geq \inf _{(Q, P) \in \mathcal{Q}_{f} \times \mathcal{P}}\left(\mathcal{H}(Q \mid P)-E^{Q}[H]\right) \\
& =\lambda\left(\mathcal{H}\left(Q^{1} \mid P^{1}\right)-E^{Q^{1}}[H]\right)+(1-\lambda)\left(\mathcal{H}\left(Q^{0} \mid P^{0}\right)-E^{Q^{0}}[H]\right) \\
& =\lambda \mathcal{H}\left(Q^{1} \mid P^{1}\right)+(1-\lambda) \mathcal{H}\left(Q^{0} \mid P^{0}\right)-E^{Q_{\lambda}}[H] \\
& =E^{P_{\lambda}}\left[\lambda \frac{d P^{1}}{d P_{\lambda}} f\left(\frac{d Q^{1}}{d P^{1}}\right)+(1-\lambda) \frac{d P^{0}}{d P_{\lambda}} f\left(\frac{d Q^{0}}{d P^{0}}\right)\right]-E^{Q_{\lambda}}[H] \\
& \geq E^{P_{\lambda}}\left[f\left(\frac{d Q_{\lambda}}{d P_{\lambda}}\right)\right]-E^{Q_{\lambda}}[H] \\
& =\mathcal{H}\left(Q_{\lambda} \mid P_{\lambda}\right)-E^{Q_{\lambda}}[H] .
\end{aligned}
$$

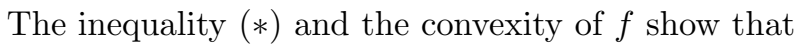

$$
\lambda \frac{d P^{1}}{d P_{\lambda}} f\left(\frac{d Q^{1}}{d P^{1}}\right)+(1-\lambda) \frac{d P^{0}}{d P_{\lambda}} f\left(\frac{d Q^{0}}{d P^{0}}\right)=f\left(\frac{d Q_{\lambda}}{d P_{\lambda}}\right) .
$$

Furthermore, since $f$ is strictly convex, this equality shows

$$
\frac{d Q^{1}}{d P^{1}}=\frac{d Q^{0}}{d P^{0}}, \quad \text { on } \quad\left\{d P^{1} / d P_{\lambda}>0, d P^{0} / d P_{\lambda}>0\right\} .
$$

The assertion (b) then follows by noting that $\left\{d P^{1} / d P_{\lambda}>0, d P^{0} / d P_{\lambda}>0\right\}=$ $\left\{d P^{0} / d P_{\lambda}>0\right\}$ if $P^{0} \ll P^{1}$.

Proof of Existence of Maximal Solution. By Lemma 4.7(b), it suffices to construct a pair $(\widehat{Q}, \widehat{P}) \in \mathcal{S}$ such that

$$
(Q, P) \in \mathcal{S} \quad \Longrightarrow \quad P \ll \widehat{P} .
$$

Let

$$
\mathcal{U}:=\left\{\{d P / d R>0\}: \exists Q \in \mathcal{Q}_{f} \text { s.t. }(Q, P) \in \mathcal{S}\right\}
$$


Note that $\mathcal{U}$ is countably additive. Indeed, if $A_{n} \in \mathcal{U}$ and $\left(Q^{n}, P^{n}\right)$ is a corresponding element of $\mathcal{S}$ for each $n,\left(\sum_{n} 2^{-n+1} Q^{n}, \sum_{n} 2^{-n+1} P^{n}\right) \in \mathcal{S}$ by Lemma 4.7 (a), and

$$
\bigcup_{n}\left\{d P^{n} / d R>0\right\}=\left\{\sum_{n} 2^{-n+1}\left(d P^{n} / d R\right)>0\right\} .
$$

Taking a sequence $\left\{A_{n}\right\} \subset \mathcal{U}$ such that

$$
R\left(A_{n}\right) \nearrow \sup _{A \in \mathcal{U}} R(A)
$$

we set $\widehat{A}:=\cup_{n} A_{n} \in \mathcal{U}$. Then we have $R(\widehat{A})=\sup _{A \in \mathcal{U}} R(A)$. $\widehat{A}$ is the essential supremum of $\mathcal{U}$ i.e.,

$$
R(A \backslash \widehat{A})=0, \quad \forall A \in \mathcal{U}
$$

Indeed, if $A \in \mathcal{U}$ and $R(A \backslash \widehat{A})>0$, then $A \cup \widehat{A} \in \mathcal{U}$ and $R(A \cup \widehat{A})=R(\widehat{A})+R(A \backslash \widehat{A})>$ $R(\widehat{A})$, hence we have a contradiction.

Let $(\widehat{Q}, \widehat{P})$ be an element corresponding to $\widehat{A}$. This pair is a desired maximal solution. Indeed, if $(Q, P) \in \mathcal{S}$, then $\{d P / d R>0\} \in \mathcal{U}$ and $R(d P / d R>0, d \widehat{P} / d R=$ $0)=0$ by (4.11). This implies $P \ll \widehat{P}$ and the proof is complete.

\subsection{Proof of Theorem 2.5}

In what follows, we fix a maximal solution $(\widehat{Q}, \widehat{P}):=\left(\widehat{Q}_{H}, \widehat{P}_{H}\right)$ to the dual problem (2.2). Note that $\widehat{Q} \ll \widehat{P}$ since $\mathcal{H}(\widehat{Q} \mid \widehat{P})<\infty$. For every $Q \in \mathcal{Q}_{f}$ (resp. $\left.P \in \mathcal{P}\right)$, we set $Q_{\lambda}:=\lambda Q+(1-\lambda) \widehat{Q}$ (resp. $P_{\lambda}:=\lambda P+(1-\lambda) \widehat{P}$ for $\lambda \in[0,1]$. Note that $Q_{\lambda} \in \mathcal{Q}_{f}$ (resp. $P_{\lambda} \in \mathcal{P}$ ) by convexity of $\mathcal{Q}_{f}$ (resp. $\mathcal{P}$ ). The next lemma is already pointed out by [12, Remark 3.10].

Lemma 4.8. For any $P \in \mathcal{P}$ and $\lambda \in[0,1), \mathcal{H}\left(\widehat{Q} \mid P_{\lambda}\right)<\infty$.

Proof. Note first that $\widehat{Q} \ll \widehat{P} \ll P_{\lambda}$, and

$$
\frac{d \widehat{Q}}{d P_{\lambda}}=\frac{d \widehat{Q} / d R}{\lambda(d P / d R)+(1-\lambda)(d \widehat{P} / d R)} \leq \frac{1}{1-\lambda} \frac{d \widehat{Q}_{H}}{d \widehat{P}} .
$$

Therefore,

$$
\begin{aligned}
\mathcal{H}\left(\widehat{Q} \mid P_{\lambda}\right) & =E^{\widehat{Q}}\left[\log \frac{d \widehat{Q}}{d P_{\lambda}}\right] \leq E^{\widehat{Q}}\left[\log \frac{1}{1-\lambda} \frac{d \widehat{Q}}{d \widehat{P}}\right] \\
& =\log \frac{1}{1-\lambda}+\mathcal{H}(\widehat{Q} \mid \widehat{P})<\infty,
\end{aligned}
$$

for every $\lambda \in[0,1)$.

We first show the joint variational inequality from which Theorem 2.5] easily follows. Define a map $f: \mathbb{R}_{+}^{2} \longrightarrow \mathbb{R} \cup\{+\infty\}$ by

$$
f(x, y)= \begin{cases}0 & \text { if } x=0 \\ +\infty & \text { if } x>0, y=0 \\ x \log (x / y) & \text { if } x>0, y>0 .\end{cases}
$$

$f$ is convex, lower semicontinuous, and $f(x, y) \geq-e^{-1} y$ for all $(x, y) \in \mathbb{R}_{+}^{2}$. Using $f$, the relative entropy is expressed as $\mathcal{H}(Q \mid P)=E^{R}[f(d Q / d R, d P / d R)]$, and $f(d Q / d R, d P / d R) \in L^{1}(R)$ if and only if $\mathcal{H}(Q \mid P)<\infty$, for every $(Q, P) \in \mathcal{Q}_{f} \times \mathcal{P}$. 
Lemma 4.9. For every $(Q, P) \in \mathcal{Q}_{f} \times \mathcal{P}$ with $\mathcal{H}(Q \mid P)<\infty$, we have

$$
R\left(\frac{d \widehat{P}}{d R}>0, \frac{d \widehat{Q}}{d R}=0, \frac{d Q}{d R}>0\right)=0 .
$$

and the random variable $\Xi(Q, P)$ defined by

$$
\begin{aligned}
\Xi(Q, P):= & 1_{\{d \widehat{P} / d R>0\}}\left\{\left(\log \frac{d \widehat{Q}}{d \widehat{P}}+1\right)\left(\frac{d Q}{d R}-\frac{d \widehat{Q}}{d R}\right)-\frac{d \widehat{Q}}{d \widehat{P}}\left(\frac{d P}{d R}-\frac{d \widehat{P}}{d R}\right)\right\} \\
& +1_{\{d \widehat{P} / d R=0, d P / d R>0\}} f\left(\frac{d Q}{d R}, \frac{d P}{d R}\right)-H\left(\frac{d Q}{d R}-\frac{d \widehat{Q}}{d R}\right)
\end{aligned}
$$

is in $L^{1}(R)$ and $E^{R}[\Xi(Q, P)] \geq 0$.

Proof. Let $(Q, P)$ be such a pair, and denote, by $Z$ (resp. $\widehat{Z}, Z_{\lambda}, D, \widehat{D}, D_{\lambda}$ ), the density of $Q$ (resp. $\widehat{Q}, Q_{\lambda}, P, \widehat{P}, P_{\lambda}$ ) w.r.t. $R$. Define $A=\{\widehat{D}>0$ or $D>0\}$ and

$$
G(\lambda):=1_{A}\left(f\left(Z_{\lambda}, D_{\lambda}\right)-Z_{\lambda} H\right), \quad \lambda \in[0,1] .
$$

$G(\lambda) \in L^{1}(R)$ and $E^{R}[G(\lambda)]=\mathcal{H}\left(Q_{\lambda} \mid P_{\lambda}\right)-E^{Q_{\lambda}}[H]$ for each $\lambda \in[0,1]$, since $f\left(Z_{\lambda}, D_{\lambda}\right)=0$ on $A^{c}=\{\widehat{D}=D=0\}$ and $H \in L^{1}\left(Q_{\lambda}\right)$ by Corollary 4.2 .

Let $\Xi(\lambda):=(G(\lambda)-G(0)) / \lambda$ for each $\lambda \in(0,1] . \Xi(\lambda) \in L^{1}(R)$ for each $\lambda \in(0,1]$, and $\Xi(\lambda)$ decreases as $\lambda \searrow 0$, since $\lambda \mapsto G(\lambda, \omega)$ is convex for a.e. $\omega$ by the convexity of $f$. Hence $\Xi(0):=\lim _{\lambda \searrow 0} \Xi(\lambda)$ is well-defined and $\Xi(0)^{+} \in L^{1}(R)$. Applying the monotone convergence theorem to the nonnegative increasing sequence $\{\Xi(1)-$ $\Xi(\lambda)\}_{\lambda}$, we have

$$
E^{R}[\Xi(0)]=\lim _{\lambda \searrow 0} E^{R}[\Xi(\lambda)] \geq 0
$$

The last inequality follows since

$$
E^{R}[\Xi(\lambda)]=\frac{\mathcal{H}\left(Q_{\lambda} \mid P_{\lambda}\right)-E^{Q_{\lambda}}[H]-\left\{\mathcal{H}(\widehat{Q} \mid \widehat{P})-E^{\widehat{Q}}[H]\right\}}{\lambda} \geq 0, \quad \forall \lambda \in(0,1],
$$

by the optimality of $(\widehat{Q}, \widehat{P})$. In particular, $E^{R}\left[\Xi(0)^{-}\right] \leq E^{R}\left[\Xi(0)^{+}\right]<\infty$, hence $\Xi(0) \in L^{1}(R)$.

Finally, we calculate the explicit form of $\Xi(0)$. Let $A_{1}:=\{\widehat{D}>0, \widehat{Z}>0\}$, $A_{2}:=\{\widehat{D}>0, \widehat{Z}=0\}$ and $A_{3}=\{\widehat{D}=0, D>0\}$. Then $A_{1}, A_{2}, A_{3}$ are disjoint and $A=A_{1} \cup A_{2} \cup A_{3}$.

On $A_{1}, D_{\lambda}>0$ and $Z_{\lambda}>0$ for every $\lambda \in[0,1]$ and $G(\lambda)=Z_{\lambda} \log \left(Z_{\lambda} / D_{\lambda}\right)-Z_{\lambda} H$. Then

$$
\Xi(0)=\left(\log \frac{\widehat{Z}}{\widehat{D}}+1-H\right)(Z-\widehat{Z})-\frac{\widehat{Z}}{\widehat{D}}(D-\widehat{D}) .
$$

On $A_{2}, Z_{\lambda}=\lambda Z$ and $G(\lambda)=\lambda Z \log \left(\lambda Z / D_{\lambda}\right)-\lambda Z H$ if $Z>0, \lambda>0$ and $G(\lambda) \equiv 0$ otherwise. Since $\lambda Z / D_{\lambda} \longrightarrow 0$ as $\lambda \searrow 0$, we have

$$
\Xi(0)= \begin{cases}-\infty & \text { if } Z>0, \\ 0 & \text { if } Z=0 .\end{cases}
$$

Since $\Xi(0) \in L^{1}(R)$, we must have $Z=0$ a.s. on $A_{2}$. This implies (4.13). 
On $A_{3}, Z_{\lambda}=\lambda Z, D_{\lambda}=\lambda D$, hence $G(\lambda)=\lambda f(Z, D)-\lambda Z H$ by the definition of $f$. Hence we get

$$
\Xi(0)=f(Z, D)-\alpha Z H=f(Z, D)-H(Z-\widehat{Z}),
$$

since $\widehat{Z}=0$. Noting that $Z=\widehat{Z}=0$ on $A^{c}$, (4.15) and (4.16) yield the expression (4.14).

Proof of Theorem 2.5. (a) By A2, there exists a pair $(Q, P) \in \mathcal{Q}_{f} \times \mathcal{P}$ such that $Q \sim P \sim R$ and $\mathcal{H}(Q \mid P)<\infty$. Applying (4.13) to this pair, we have

$$
R\left(\frac{d \widehat{P}}{d R}>0, \frac{d \widehat{Q}}{d R}=0\right)=R\left(\frac{d \widehat{P}}{d R}>0, \frac{d \widehat{Q}}{d R}=0, \frac{d Q}{d R}>0\right)=0 .
$$

This implies that $\widehat{P} \ll \widehat{Q}$. Since $\widehat{Q} \ll \widehat{P}$ by assumption, we have $\widehat{Q} \sim \widehat{P}$.

(b) Let $Q \in \mathcal{Q}_{f}$ be such that $\mathcal{H}(Q \mid \widehat{P})<\infty$. Then we can apply Lemma 4.9 to the pair $(Q, \widehat{P})$. In this case,

$$
\Xi(Q, \widehat{P})=\left(\log \frac{d \widehat{Q}}{d \widehat{P}}+1-H\right)\left(\frac{d Q}{d R}-\frac{d \widehat{Q}}{d R}\right), \quad R \text {-a.s. }
$$

Since $\log \frac{d \widehat{Q}}{d \widehat{P}}-\alpha H \in L^{1}(\widehat{Q})$ and $\Xi(Q, \widehat{P}) \in L^{1}(R)$, we have

$$
\begin{aligned}
E^{Q}\left[\left|\log \frac{d \widehat{Q}}{d \widehat{P}}-H\right|\right] & =E^{R}\left[\left|\frac{d Q}{d R}\left(\log \frac{d \widehat{Q}}{d \widehat{P}}-H\right)\right|\right] \\
& \leq E^{R}[|\Xi(Q, \widehat{P})|]+2+E^{\widehat{Q}}\left[\left|\log \frac{d \widehat{Q}}{d \widehat{P}}-H\right|\right]<\infty .
\end{aligned}
$$

Then (4.14) yields that

$$
0 \leq E^{R}[\Xi(Q, \widehat{P})]=E^{Q}\left[\log \frac{d \widehat{Q}}{d \widehat{P}}-H\right]-E^{\widehat{Q}}\left[\log \frac{d \widehat{Q}}{d \widehat{P}}-H\right]
$$

This implies (2.6).

(c) By Lemma 4.8, we can apply Lemma 4.9 to $(\widehat{Q}, P)$ for every $P \in \mathcal{P}$. Noting that $f(d \widehat{Q} / d R, d P / d R)=0$ on $\{d \widehat{P} / d R=0\}$, we have

$$
\Xi(\widehat{Q}, P)=-\frac{d \widehat{Q}}{d \widehat{P}}\left(\frac{d P}{d R}-\frac{d \widehat{P}}{d R}\right)
$$

and (2.7) follows.

\section{Proofs of Theorem 2.8 and 2.9}

We first prove Theorem 2.8, using the following version of minimax theorem due to Fan [1]. A simple proof is found in Simons 37.

Theorem 5.1 (Fan [1]). Let $A$ be a convex subset of a linear space, $B$ a compact convex subset of a Hausdorff space, and $f$ is a real valued function on $A \times B$. Suppose that

(1) for each $x \in A, y \mapsto f(x, y)$ on $B$ is concave and upper semicontinuous,

(2) for each $y \in B, x \mapsto f(x, y)$ on $A$ is convex.

Then

$$
\inf _{x \in A} \sup _{y \in B} f(x, y)=\sup _{y \in B} \inf _{x \in A} f(x, y)
$$


Lemma 5.2. Under $\boldsymbol{A} \mathbf{1}-\boldsymbol{A} 3$, it holds that

$$
\inf _{\theta \in \Theta_{b}} \sup _{P \in \mathcal{P}} E^{P}\left[e^{-\alpha\left(\theta \cdot S_{T}-H\right)}\right]=\sup _{P \in \mathcal{P}} \inf _{\theta \in \Theta_{b}} E^{P}\left[e^{-\alpha\left(\theta \cdot S_{T}-H\right)}\right] .
$$

Proof. Let

$$
\mathcal{Z}:=\left\{e^{\alpha H} d P / d R: P \in \mathcal{P}\right\} .
$$

Then the claim is equivalent to saying that

$$
\inf _{\theta \in \Theta_{b}} \sup _{Z \in \mathcal{Z}} E^{R}\left[Z e^{-\alpha \theta \cdot S_{T}}\right]=\sup _{Z \in \mathcal{Z}} \inf _{\theta \in \Theta_{b}} E^{R}\left[Z e^{-\alpha \theta \cdot S_{T}}\right] .
$$

We want to apply Theorem 5.1 to the map $\Theta_{b} \times \mathcal{Z} \ni(\theta, Z) \mapsto E^{R}\left[Z e^{-\alpha \theta \cdot S_{T}}\right]$. To do this, we check the assumptions of Theorem 5.1 .

First, $\mathcal{Z}$ is convex and weakly compact. Indeed, the convexity follows immediately from $\mathbf{A} \mathbf{1}$, and $\mathcal{Z}$ is uniformly integrable by $\mathbf{A 3}$. Let $\left\{Z_{n}\right\}$ be a Cauchy sequence of $\mathcal{Z}$ as a subset of $L^{1}(R)$, with $Z_{n} \longrightarrow Z$ in $L^{1}(R)$ for some $Z \in L^{1}(R)$. There then exists $P_{n} \in \mathcal{P}$ such that $Z_{n}=e^{\alpha H} d P_{n} / d R$ for each $n$. Taking a subsequence $\left\{Z_{n_{m}}\right\}$, if necessary, we have $Z_{n_{m}} \longrightarrow Z$ a.s., showing that

$$
\frac{d P_{n_{m}}}{d R} \longrightarrow e^{-\alpha H} Z, \quad \text { a.s. }
$$

Since the set $\{d P / d R: P \in \mathcal{P}\}$ is uniformly integrable and closed by $\mathbf{A 1}$, this convergence takes place in $L^{1}(R)$, and $d P / d R:=e^{-\alpha H} Z$ defines a probability measure in $\mathcal{P}$, hence $Z \in \mathcal{Z}$. Since $\left\{Z_{n}\right\}$ is a Cauchy sequence, we have $\| Z_{n}-$ $Z\left\|_{L^{1}} \leq\right\| Z_{n}-Z_{n_{m}}\left\|_{L^{1}}+\right\| Z_{n_{m}}-Z \|_{L^{1}} \longrightarrow 0$. Hence $\mathcal{Z}$ is weakly compact by the Dunford-Pettis theorem [9, Theorem II.25].

Next, the map $Z \mapsto E^{R}\left[Z e^{-\alpha \theta \cdot S_{T}}\right]$ is a (strongly) continuous linear functional on $L^{1}(R)$ for each $\theta \in \Theta_{b}$, since $\theta \cdot S_{T}$ is bounded by definition. In particular, this map is even weakly continuous by the definition of the weak topology.

Finally, $\Theta_{b}$ is a linear, hence convex, subset of a linear space $L(S, R)$, and the $\operatorname{map} \theta \mapsto E^{R}\left[Z e^{-\alpha \theta \cdot S_{T}}\right]$ is convex for each $Z \in \mathcal{Z}$. Therefore, we can apply Theorem 5.1. and the proof is complete.

For fixed $P \in \mathcal{P}$, the problem

$$
\text { minimize } E^{P}\left[e^{-\alpha\left(\theta \cdot S_{T}-H\right)}\right], \quad \text { over all } \theta \in \Theta_{b}
$$

is the classical exponential hedging problem, for which the duality equality is shown by [1] (see also [7]). However, not all $P \in \mathcal{P}$ satisfy the assumptions of this result, hence we need to replace $\mathcal{P}$ by a subset for which the classical duality equality holds, and the value of the problem remains unchanged.

By A2, there exists a pair $(\bar{Q}, \bar{P}) \in \mathcal{Q}_{f} \times \mathcal{P}$ such that $\bar{Q} \sim \bar{P} \sim R$ and $\mathcal{H}(\bar{Q} \mid \bar{P})<$ $\infty$. We fix such a pair, and set $P_{\lambda}:=\lambda \bar{P}+(1-\lambda) P$ for each $P \in \mathcal{P}$. Then define

$$
\overline{\mathcal{P}}:=\left\{P_{\lambda}: P \in \mathcal{P}, \lambda \in(0,1]\right\} .
$$

LEMMA 5.3.

$$
\sup _{P \in \mathcal{P}} \inf _{\theta \in \Theta_{b}} E^{P}\left[e^{-\alpha\left(\theta \cdot S_{T}-H\right)}\right]=\sup _{P \in \overline{\mathcal{P}}} \inf _{\theta \in \Theta_{b}} E^{P}\left[e^{-\alpha\left(\theta \cdot S_{T}-H\right)}\right] .
$$

Proof. Let $P \in \mathcal{P}$ be fixed. Since

$$
E^{P_{\lambda}}\left[e^{-\alpha\left(\theta \cdot S_{T}-H\right)}\right]=\lambda E^{\bar{P}}\left[e^{-\alpha\left(\theta \cdot S_{T}-H\right)}\right]+(1-\lambda) E^{P}\left[e^{-\alpha\left(\theta \cdot S_{T}-H\right)}\right],
$$


the map $\lambda \mapsto E^{P_{\lambda}}\left[e^{-\alpha\left(\theta \cdot S_{T}-H\right)}\right]$ is affine on $[0,1]$, for each $\theta \in \Theta_{b}$. Hence $\lambda \mapsto$ $\inf _{\theta \in \Theta_{b}} E^{P_{\lambda}}\left[e^{-\alpha\left(\theta \cdot S_{T}-H\right)}\right]$ is concave, and finite valued on $[0,1]$ since $e^{-\alpha\left(\theta \cdot S_{T}-H\right)}$ is bounded from below, $0 \in \Theta_{b}$ and $e^{\alpha H} \in L^{1}\left(P_{\lambda}\right)$ by A3. In particular, this map is lower semicontinuous, hence

$$
\inf _{\theta \in \Theta_{b}} E^{P}\left[e^{-\alpha\left(\theta \cdot S_{T}-H\right)}\right] \leq \sup _{\lambda \in(0,1]} \inf _{\theta \in \Theta_{b}} E^{P_{\lambda}}\left[e^{-\alpha\left(\theta \cdot S_{T}-H\right)}\right] .
$$

This implies that

$$
\begin{aligned}
\sup _{P \in \mathcal{P}} \inf _{\theta \in \Theta_{b}} E^{P}\left[e^{-\alpha\left(\theta \cdot S_{T}-H\right)}\right] & \leq \sup _{P \in \mathcal{P}} \sup _{\lambda \in(0,1]} \inf _{\theta \in \Theta_{b}} E^{P_{\lambda}}\left[e^{-\alpha\left(\theta \cdot S_{T}-H\right)}\right] \\
& =\sup _{P \in \overline{\mathcal{P}}} \inf _{\theta \in \Theta_{b}} E^{P}\left[e^{-\alpha\left(\theta \cdot S_{T}-H\right)}\right] .
\end{aligned}
$$

The converse inequality is trivial since $\overline{\mathcal{P}} \subset \mathcal{P}$, and the proof is complete.

Proof of Theorem 2.8, By Lemma 5.2 and Lemma 5.3, it suffices to show that

$$
\sup _{P \in \overline{\mathcal{P}}} \inf _{\theta \in \Theta_{b}} E^{P}\left[e^{-\alpha\left(\theta \cdot S_{T}-H\right)}\right]=e^{-\inf _{(Q, P) \in \mathcal{Q}_{f} \times \mathcal{P}}\left(\mathcal{H}(Q \mid P)-\alpha E^{Q}[H]\right)} .
$$

Note that $P \sim \bar{Q} \sim R$ and $\mathcal{H}(\bar{Q} \mid P)<\infty$ for every $P \in \overline{\mathcal{P}}$ by Lemma 4.8. Also, A3 implies for every $P \in \overline{\mathcal{P}}$ that

$$
E^{P}\left[e^{(\alpha+\varepsilon)|H|}\right]<\infty, \quad \exists \varepsilon>0 .
$$

Hence the assumptions of [22, Theorem 2.1] (see also [7, Theorem 2.3]) are satisfied under each $P \in \overline{\mathcal{P}}$, hence we have

$$
\inf _{\theta \in \Theta_{b}} E^{P}\left[e^{-\alpha\left(\theta \cdot S_{T}-H\right)}\right]=e^{-\inf _{Q \in \mathcal{Q}_{f}(P)}\left(\mathcal{H}(Q \mid P)-\alpha E^{Q}[H]\right)}, \quad \forall P \in \overline{\mathcal{P}},
$$

where $\mathcal{Q}_{f}(P):=\left\{Q \in \mathcal{Q}_{f}: \mathcal{H}(Q \mid P)<\infty\right\}$. Taking the supremum in $P \in \overline{\mathcal{P}}$

$$
\sup _{P \in \overline{\mathcal{P}}} \inf _{\theta \in \Theta_{b}} E^{P}\left[e^{-\alpha\left(\theta \cdot S_{T}-H\right)}\right]=e^{-\inf _{P \in \overline{\mathcal{P}}} \inf _{Q \in \mathcal{Q}_{f}(P)}\left(\mathcal{H}(Q \mid P)-\alpha E^{Q}[H]\right)} .
$$

It remains to show that

$$
\inf _{P \in \overline{\mathcal{P}}} \inf _{Q \in \mathcal{Q}_{f}(P)}\left(\mathcal{H}(Q \mid P)-\alpha E^{Q}[H]\right)=\inf _{(Q, P) \in \mathcal{Q}_{f} \times \mathcal{P}}\left(\mathcal{H}(Q \mid P)-\alpha E^{Q}[H]\right) .
$$

Let $P \in \mathcal{P}$ and $Q \in \mathcal{Q}_{f}(P)$. Then $\lambda \mapsto \mathcal{H}\left(Q \mid P_{\lambda}\right)-\alpha E^{Q}[H]$ is convex and finite valued on $[0,1)$ (Lemma 4.8), hence upper semicontinuous at $\lambda=0$. Thus, the lower envelope $\lambda \mapsto \inf _{Q \in \mathcal{Q}_{f}(P)}\left(\mathcal{H}\left(Q \mid P_{\lambda}\right)-\alpha E^{Q}[H]\right)$ is also upper semicontinuous at $\lambda=0$. This yields that

$$
\inf _{Q \in \mathcal{Q}_{f}(P)}\left(\mathcal{H}(Q \mid P)-\alpha E^{Q}[H]\right) \geq \inf _{\lambda \in(0,1]} \inf _{Q \in \mathcal{Q}_{f}(P)}\left(\mathcal{H}\left(Q \mid P_{\lambda}\right)-\alpha E^{Q}[H]\right),
$$

for each $P \in \mathcal{P}$. Taking the infimum in $P \in \mathcal{P}$.

$$
\begin{aligned}
\inf _{P \in \mathcal{P}} \inf _{Q \in \mathcal{Q}_{f}(P)}\left(\mathcal{H}(Q \mid P)-\alpha E^{Q}[H]\right) & \geq \inf _{P \in \mathcal{P}} \inf _{\lambda \in(0,1]} \inf _{Q \in \mathcal{Q}_{f}(P)}\left(\mathcal{H}\left(Q \mid P_{\lambda}\right)-\alpha E^{Q}[H]\right) \\
& =\inf _{P \in \overline{\mathcal{P}}} \inf _{Q \in \mathcal{Q}_{f}(P)}\left(\mathcal{H}(Q \mid P)-\alpha E^{Q}[H]\right) .
\end{aligned}
$$

Noting that $\mathcal{H}(Q \mid P)-\alpha E^{Q}[H]=+\infty$ if $Q \in \mathcal{Q}_{f} \backslash \mathcal{Q}_{f}(P)$ by Corollary 4.2, we have

$$
\inf _{(Q, P) \in \mathcal{Q}_{f} \times \mathcal{P}}\left(\mathcal{H}(Q \mid P)-\alpha E^{Q}[H]\right) \geq \inf _{P \in \overline{\mathcal{P}}} \inf _{Q \in \mathcal{Q}_{f}}\left(\mathcal{H}(Q \mid P)-\alpha E^{Q}[H]\right) .
$$

The converse inequality is trivial since $\overline{\mathcal{P}} \subset \mathcal{P}$, and we get (5.7). 
Before giving the proof of Theorem 2.9, we recall some basic facts from convex analysis. Let $f: \mathcal{X} \times \mathcal{Y} \longrightarrow \mathbb{R} \cup\{ \pm \infty\}$, where $\mathcal{X}$ and $\mathcal{Y}$ are arbitrary nonempty sets. A point $(\widehat{x}, \widehat{y}) \in \mathcal{X} \times \mathcal{Y}$ is said to be a saddle point of $f$ if

$$
\min _{x \in \mathcal{X}} f(x, \widehat{y})=f(\widehat{x}, \widehat{y})=\sup _{y \in \mathcal{Y}} f(\widehat{x}, y) .
$$

Then the saddle point theorem states that a pair $(\widehat{x}, \widehat{y}) \in \mathcal{X} \times \mathcal{Y}$ is a saddle point of $f$ if and only if

$$
\widehat{x} \in \arg \inf _{\mathcal{X}}\left(\sup _{y \in \mathcal{Y}} f(\cdot, y)\right), \quad \widehat{y} \in \arg \sup _{\mathcal{Y}}\left(\inf _{x \in \mathcal{X}} f(x, \cdot)\right)
$$

and the following minimax equality holds:

$$
\inf _{x \in \mathcal{X}} \sup _{y \in \mathcal{Y}} f(x, y)=\sup _{y \in \mathcal{Y}} \inf _{x \in \mathcal{X}} f(x, y) .
$$

Here the inequality " $\geq$ " is always true (minimax inequality). See [30] for detail. It is worth noting that the above argument does not require any topological properties of $\mathcal{X}, \mathcal{Y}$ and $f$.

Proof of Theorem 2.9. (a) Let $\theta \in \Theta_{H}$. Then in particular, $\theta \cdot S$ is a martingale under $\widehat{Q}_{H}$. Therefore, noting that $\widehat{Q}_{H} \sim \widehat{P}_{H}$ by Theorem 2.5, and using (2.8), we have

$$
\begin{aligned}
\sup _{P \in \mathcal{P}} E^{P}\left[e^{-\alpha\left(\theta \cdot S_{T}-H\right)}\right] & \geq E^{\widehat{P}_{H}}\left[e^{-\alpha\left(\theta \cdot S_{T}-H\right)}\right] \\
& =E^{\widehat{Q}_{H}}\left[\frac{d \widehat{P}_{H}}{d \widehat{Q}_{H}} e^{-\alpha\left(\theta \cdot S_{T}-H\right)}\right] \\
& =E^{\widehat{Q}_{H}}\left[e^{-\alpha\left(\theta \cdot S_{T}-\hat{\theta} \cdot S_{T}\right)}\right] \cdot E^{\widehat{P}_{H}}\left[e^{-\alpha\left(\hat{\theta} \cdot S_{T}-H\right)}\right] \\
& \geq e^{-\alpha E^{\widehat{Q}_{H}}\left[\theta \cdot S_{T}-\hat{\theta} \cdot S_{T}\right]} \cdot E^{\widehat{P}_{H}}\left[e^{-\alpha\left(\hat{\theta} \cdot S_{T}-H\right)}\right] \\
& \geq E^{\widehat{P}_{H}}\left[e^{-\alpha\left(\hat{\theta} \cdot S_{T}-H\right)}\right]
\end{aligned}
$$

Here the fourth inequality follows from Jensen's inequality, while the fifth from the $\widehat{Q}_{H}$-martingale property of $\theta \cdot S$ and $\hat{\theta} \cdot S$. On the other hand, taking the logarithm in (2.8), and using again the $\widehat{Q}_{H}$-martingale property of $\hat{\theta} \cdot S$, we have

$$
\begin{aligned}
E^{\widehat{P}_{H}}\left[e^{-\alpha\left(\hat{\theta} \cdot S_{T}-H\right)}\right] & =e^{-\left(\mathcal{H}\left(\widehat{Q}_{H} \mid \widehat{P}_{H}\right)-\alpha E^{\widehat{Q}_{H}}[H]\right)} \\
& =e^{-\inf _{(Q, P) \in \mathcal{Q}_{f} \times \mathcal{P}\left(\mathcal{H}(Q \mid P)-\alpha E^{Q}[H]\right)}} .
\end{aligned}
$$

Combining (5.10) and (5.11), we have

$$
\inf _{\theta \in \Theta_{H}} \sup _{P \in \mathcal{P}} E^{P}\left[e^{-\alpha\left(\theta \cdot S_{T}-H\right)}\right] \geq e^{-\inf _{(Q, P) \in \mathcal{Q}_{f} \times \mathcal{P}}\left(\mathcal{H}(Q \mid P)-\alpha E^{Q}[H]\right)} .
$$

The converse inequality follows from Theorem 2.8 and the inclusion $\Theta_{b} \subset \Theta_{H}$, and we get (2.12).

(b) If $\widehat{Q}_{H} \sim R, \hat{\theta} \in L(S, R)$. Also, $\hat{\theta} \cdot S$ is a martingale under each $Q \in \mathcal{Q}_{f}\left(\widehat{P}_{H}\right)$ by [22, Theorem $2.1(\mathrm{~b})]$. Hence $\hat{\theta} \in \Theta_{H}$. We shall prove that the pair $\left(\hat{\theta}, \widehat{P}_{H}\right)$ is a saddle point of $(\theta, P) \mapsto E^{P}\left[e^{-\alpha\left(\theta \cdot S_{T}-H\right)}\right]$, on $\Theta_{H} \times \mathcal{P}$.

First, (5.10) shows that

$$
E^{\widehat{P}_{H}}\left[e^{-\alpha\left(\hat{\theta} \cdot S_{T}-H\right)}\right]=\inf _{\theta \in \Theta_{H}} E^{\widehat{P}_{H}}\left[e^{-\alpha\left(\theta \cdot S_{T}-H\right)}\right] .
$$


On the other hand, since the representation (2.8) holds under every $P \in \mathcal{P}$ by $\mathbf{A 4}$, the variational inequality (2.7) shows that

$$
\begin{aligned}
E^{P}\left[e^{-\alpha\left(\hat{\theta} \cdot S_{T}-H\right)}\right] & =E^{P}\left[\frac{d \widehat{Q}_{H}}{d \widehat{P}_{H}}\right] \cdot E^{\widehat{P}_{H}}\left[e^{-\alpha\left(\hat{\theta} \cdot S_{T}-H\right)}\right] \\
& \leq E^{\widehat{P}_{H}}\left[e^{-\alpha\left(\hat{\theta} \cdot S_{T}-H\right)}\right]
\end{aligned}
$$

for every $P \in \mathcal{P}$. Hence we have that $\left(\hat{\theta}, \widehat{P}_{H}\right) \in \Theta_{H} \times \mathcal{P}$ is a saddle point.

Finally, the saddle point theorem shows that $\hat{\theta}$ is optimal, $\widehat{P}_{H}$ is least favorable, and the minimax equality (2.14) holds.

REMARK 5.4. In the proof of (5.10), $\widehat{Q}_{H}$-martingale property of $\theta \cdot S$ is not actually necessary, and the $\widehat{Q}_{H}$-supermartingale property is sufficient. In particular, Theorem 2.9 remains true even if we replace $\Theta_{H}$ by another $\Theta$ whenever $\Theta$ contains $\Theta_{b} \cup\{\hat{\theta}\}$ and $\theta \cdot S$ is a $\widehat{Q}_{H}$-supermartingale for every $\theta \in \Theta$. In this sense, we can say that the strategy $\hat{\theta}$ is optimal whenever it is admitted.

\section{REFERENCES}

[1] J.-P. Ansel and C. Stricker (1994). Couverture des actifs contingents et prix maximum. Ann. Inst. Henri Princaré, 30(2):303-315.

[2] M. Avellaneda, M. Levy, and A. Paras (1995). Pricing and hedging derivative sequrities in markets with uncertain volatilities. Appl. Math. Finance, 2:73-88.

[3] D. Becherer (2003). Rational hedging and valuation of integrated risks under constant absolute risk aversion. Insurance Math. Econom., 33(1-28).

[4] G. Bordigoni, A. Matoussi, and M. Schweizer (2007). A stochastic control approach to a robust utility maximization problem. In F. E. Benth, G. Di Nunno, T. Lindstrom, B. Oksendal, and T. Zhang, editors, Stochastic Analysis and Applications. Proceedings of the Second Abel Symposium, Oslo, 2005, pages 125-151. Springer-Verlag.

[5] I. Csizár (1975). I-divergence geometry of probability distributions and minimization problems. Ann. Probab., 3(1):146-158.

[6] M. H. A. Davis (2006). Optimal hedging with basis risk. In Y. Kabanov, R. Liptser, and J. Stoyanov, editors, From Stochastic Calculus to Mathematical Finance, pages 169-187. Springer-Verlag.

[7] F. Delbaen, P. Grandits, T. Rheinländer, D. Samperi, M. Schweizer, and C. Stricker (2002). Exponential hedging and entropic penalties. Math. Finance, $12(2): 99-123$.

[8] F. Delbaen and W. Schachermayer (1994). A general version of the fundamental theorem of asset pricing. Math. Ann., 300:463-520.

[9] C. Dellacherie and P.-A. Meyer (1978). Probabilities and Potential A. NorthHolland Mathematics Studies, vol. 29. North-Holland.

[10] L. Denis and C. Martini (2006). A theoretical framework for the pricing of contingent claims in the presense of model uncertainty. Ann. Appl. Probab., $16(2): 827-852$.

[11] K. Fan (1953). Minimax theorems. Proc. Nat. Acad. Sci. U.S.A, 39:42-47.

[12] H. Föllmer and A. Gundel (2006). Robust projections in the class of martingale measures. Illinois J. Math., 50(2):439-472. 
[13] H. Föllmer and A. Schied (2004). Stochastic Finance. De Gruyter Studies in Mathematics, vol. 27. Walter de Gruyter, 2nd edition.

[14] H. Föllmer, A. Schied, and S. Weber (2007). Robust preferences and robust portfolio choice. Preprint.

[15] M. Frittelli (2000). The minimal entropy martingale measure and the valuation problem in incomplete markets. Math. Finance, 10(1):39-52.

[16] T. Goll and L. Rüschendorf (2001). Minimax and minimal distance martingale measures and their relationship to portfolio optimization. Finance Stoch., 5 (4):557-581.

[17] P. Grandits and T. Rheinländer (2002). On the minimal entropy martingale measure. Ann. Probab., 30(3):1003-1038.

[18] A. Gundel (2005). Robust utility maximization for complete and incomplete market models. Finance Stoch., 9(2):151-176.

[19] D. Hernández-Hernández and A. Schied (2006). Robust utility maximization in a stochastic factor model. Statist. Decisions, 24(3):109-125.

[20] D. Hernández-Hernández and A. Schied (2007). A control approach to robust ulility maximization with logarithmic utility and time-consistent penalties. Stochastic Process. Appl., 117:980-1000.

[21] S. D. Hodges and A. Neuberger (1989). Optimal replication of contingent claims under transaction costs. Review of Futures Markets, 8:222-239.

[22] Y. M. Kabanov and C. Stricker (2002). On the optimal portfolio for the exponential utility maximization: remarks to the six-author paper. Math. Finance, 12(2):125-134.

[23] D. Kramkov and W. Schachermayer (1999). The asymptotic elasticity of utility functions and optimal investment in incomplete markets. Ann. Appl. Probab., 9(3):904-950.

[24] D. Kramkov and W. Schachermayer (2003). Necessary and sufficient conditions in the problem of optimal investment in incomplete markets. Ann. Appl. Probab., 13(4):1504-1516.

[25] M. Mania and M. Schweizer (2005). Dynamic exponential utility indifference valuation. Ann. Appl. Probab., 15(3):2113-2143.

[26] Y. Miyahara (1996). Canonical martingale measures of incomplete assets markets. In S. Watanabe, M. Fukushima, Y. V. Prohorov, and Y. N. Shiriyaev, editors, Probability Theory and Mathematical Statistics: Proceedings of the Seventh Japan-Russia Symposium, Tokyo, pages 343-352. World Scientific.

[27] M. Müller (2005). Market Completion and Robust Utility Maximization. PhD thesis, Humboldt Universität zu Berlin.

[28] M. Musiela and T. Zariphopoulou (2004). An example of indifference prices under exponential preferences. Finance Stoch., 8(229-239).

[29] M.-C. Quenez (2004). Optimal portfolio in a multiple-priors model. In R. C. Dalang, M. Dozzi, and F. Russo, editors, Seminar on Stochastic Analysis, Random Fields and Applications IV, Progress in Probability, vol. 58, pages 291-321. Birkhäuser Verlag.

[30] R. T. Rockafellar (1970). Convex Analysis. Princeton University Press.

[31] R. Rouge and N. El Karoui (2000). Pricing via utility maximization and entropy. Math. Finance, 10(2):259-276.

[32] W. Schachermayer (2001). Optimal investment in incomplete markets when wealth may become negative. Ann. Appl. Probab., 11(3):694-734. 
[33] W. Schachermayer (2003). A super-martingale property of the optimal portfolio process. Finance Stoch., 7(4):433-456.

[34] A. Schied (2007). Optimal investment for risk- and ambiguity-averse preferences: a duality approach. Finance Stoch., 11(1):107-129.

[35] A. Schied and C.-T. Wu (2005). Duality theory for optimal investment under model uncertainty. Statist. Decisions, 23(3):199-217.

[36] J. Sekine (2006). On exponential hedging and related quadratic backward stochastic differential equations. Appl. Math. Optim., 54:131-158.

[37] S. Simons (1998). Minimax and Monotonicity. Lecture Notes in Math., vol. 1693. Springer-Verlag.

[38] M. Yor (1978). Sous-espaces denses dans $L^{1}$ ou $H^{1}$ et representation des martingales. In Séminaire de Probabilités de Strasbourg XII, Lecture Notes in Math., vol. 649, pages 265-309. Springer-Verlag. 